\title{
Effect of RACH Procedure on the Performance of LTE- based M2M Communication
}

\author{
A. I. A. Jabbar, PhD \\ Engineering College \\ Department of Electrical Engineering \\ Mosul University
}

\author{
Fawaz Y. Abdullah \\ Engineering College \\ Department of Electrical Engineering \\ Mosul University
}

\begin{abstract}
In this paper, the parameters of the Random Access Channel (RACH) of LTE/ LTE-A based M2M communication under congestion situation are investigated. These parameters include the effect of the Backoff Indicator (BI), Hybrid Automatic Repeat-reQuest (HARQ) and contention resolution timer (CRT) on the Performance of LTE-based M2M Communication. The results indicate that the parameters of the RACH procedure have an impact on the network's performance, such as delay and packets dropped.
\end{abstract}

\section{General Terms}

Computer Network, Wireless Networks, M2M, RACH procedure

\section{Keywords}

M2M, LTE networks, RACH procedure, PRACH..

\section{INTRODUCTION}

Third Generation Partnership Project (3GPP) and other different cellular network standardization organizations have started working on developing global standards for supporting Machine-to-Machine (M2M) over existing mobile networks. LTE Advanced is the first wireless system to standardize the constraint of latency and traffic policies for M2M applications.

3GPP has identified Random Access Channel (RACH) overload as the critical problem in cellular M2M. RACH is the first channel initially used by Mobile Station (User Equipment (UE) in LTE) or M2M device to the accessed cellular network (from $2 \mathrm{G}$ through to $\mathrm{LTE}(4 \mathrm{G})$ ). Also, $5 \mathrm{G}$ networks are expected to have similar RACH structure and access techniques to its predecessors. RACH, as the first link to the cellular network, is an imperative channel which is organized into frames and slots where access attempts are only allowed in slots. Therefore despite its poor throughput performance, traditional slotted ALOHA (s-ALOHA) has been the popular random access scheme to use for the RACH access in all cellular network standards.

In this paper, we focus on the problem of congestion and system overload in M2M applications over LTE cellular networks. This issue happens when the massive amount of M2M devices try to access the network, sending signaling at the same time. Take Smart Electric Metering (SEM) application as an example; meters periodically report the electric power usage to the server for billing application[1]. When these meters try to transmit their data almost at the same time, the maximum load situations have a tremendous impact on the operations of the LTE network, and both M2M and non-M2M traffic could be affected[2]. Therefore, aiming to solve the problem of congestion, the $3 \mathrm{GPP}$ proposed the following schemes:

1) Introducing new barring factors for $\mathrm{M} 2 \mathrm{M}$ equipment to avoid network congestion and control access,

2) Classifying $\mathrm{RACH}$ resources,

3) Setting specific back-off time for M2M equipment,

4) Allocating specific slot forM2Mequipments to access, and so on.

Ming-Yuan Cheng et al. [3] implemented Overload Control for Machine Type Communications (MTC) in the LTEAdvanced NETWORK and improved the performance. They RACH overload because a huge number of M2M devices want to use RACH resources. The negative impact of $\mathrm{M} 2 \mathrm{M}$ collision is $\mathrm{RACH}$ procedure failure. On the other hand, this problem is solved by four methods: the Push Base Method [3]; Randomized Access Dispersion; Backoff Indicator Adjustment; and the P-Persistent Approach.

Manowar and Akram [4] introduced RA for M2M Communication in LTE-A Networks: Issues and Approaches. This research focuses on RACH overload when a huge number of M2Ms attempt to access the network, leading to high network congestion.

Dimas and Kae [5] presented Hybrid RA and Data Transmission Protocol for M2M Communications in Mobile Networks. They assume that RACH may be congested if a large number of M2M devices attempt to access the network simultaneously. The hybrid RA is introduced to solve this issue [5].

No related research shows the effect of all the parameters of the RACH procedure on the LTE network. In this paper, the effect of the Backoff Indicator (BI), ), Hybrid Automatic Repeat-reQuest (HARQ) and contention resolution timer (CRT) on the performance of LTE-based M2M system is studied. It aims to reach the optimum performance of there parameters.

The remainder of the paper is organized as follows. Section two, the random access procedure in LTE is described, and issues related to massive access are briefly explained. The simulation analysis and results presented in Sections 3 and 4. Finally, conclusions are drawn in Section five. 


\section{LTE-A M2M STANDARD AND RACH PROCEDURES}

This section explains the contention-based Random Access (RA) procedure defined for LTE networks[6][ 7][ 8][ 9]. The RA procedure mainly consists of a four-message handshake between the device (UE) and the eNodeB. Fig. 1 illustrates the LTE RA procedure with the following steps:

1) Transmitting Random Access Preamble.

2) Receiving Random Access Response.

3) Transmitting RRC Connection Request.

4) Receiving Contention Resolution.

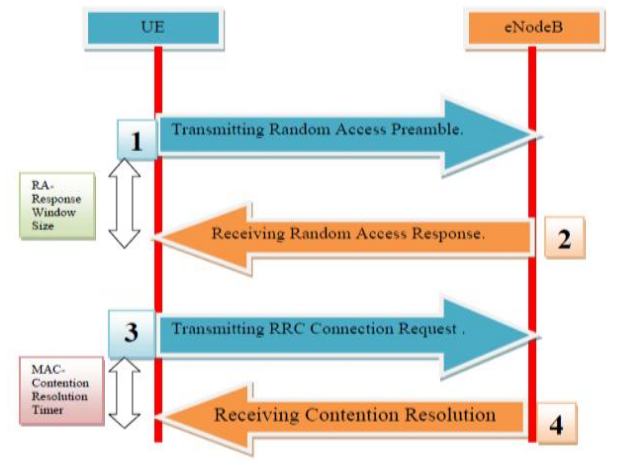

Figure 1. LTE Random Access Procedure

\subsection{Transmitting Random-Access Preamble (Message1)}

A device attempting to establish a connection transmits a randomly selected preamble (chosen among a set of a maximum of 64 possible preambles, even though some of them may be reserved for prioritized access) in the next available RACH resource. The eNodeB can estimate the transmission time of the device by detecting its randomaccess preamble. Since it is possible that multiple devices send preambles simultaneously, there may be collisions during the access procedure[10]. These collisions will be detected in Message 3, as explained later.

\subsection{Receiving Random-Access Response (Message2)}

For each detected preamble in each accessed resource (slot), the eNodeB sends a time advance command to all the devices which transmitted a specific preamble in a specific PRACH to adjust synchronization. Also, the eNodeB allocates transmission resources to the devices which sent a given preamble in a given PRACH for the transmission of Message 3. If a device sends Message 1 and it does not receive the RAR from the eNodeB in a period, called $\boldsymbol{R} A$ Response Window Size (RAR), or it receives an RAR which does not attach information related to its access request, it postpones the access attempt to the next RACH opportunity. It can happen due to either a collision or a channel fading which has corrupted the transmitted RAR. The MAC header may carry the backoff parameter values, denoted as Backoff Indicator (BI), for the collided or undetected UEs [6]. The collided or undetected devices should wait for a specific number of sub-frames before it attempts to access the channel again. The backoff counter expresses the number of sub-frames. If a device receives the RAR without information that the preamble it selected and transmitted in the Msg1, the device randomly chooses a backoff counter from zero to the BI and retransmits a newly selected $\boldsymbol{R A}$ preamble (Msg1) in the next available RA slot when the backoff counter expires (i.e., decreases to zero). In LTE, the range of $\mathrm{BI}$ is from 0 to 960 sub-frames [6][8]. The procedure continues until the maximum number of preamble transmissions is reached. If the max. number of transmissions is reached, additional attempts are blocked.

\subsection{Radio Resource Control (RRC) Connection Request (Message 3):}

The device which sent Message 1 to initiate the access procedure and receives the RAR associated to its transmitted preamble transmits, in the assigned resources notified in the RAR, its temporary terminal identity to the eNB using the Physical Uplink Shared Channel (PUSCH) to request an RRC connection. In the case that two or more MD users sent Message 1 using the same preamble in the same PRACH (Fig.2), and the collision was not detected in Message 2 (due to a constructive interference), Message 3, will collide. To detect such collision, Message 3 is transmitted with Hybrid Automatic Repeat-reQuest (HARQ). Upon a maximum number of attempts to send Message 3, a collision is declared, and access to the system is postponed.

\subsection{RRC Connection Setup (Message 4 ):}

The eNodeB sends information allocating resources to each of the devices which gained access, specifying their addresses. Therefore, the connection is established, and the device can start the transmission of data. If the eNodeB does not receive the ACK for the Message 4, it waits for a gap time for the Message 4 retransmission. The number of HARQ retransmission of the Message 3 and the Message 4 is limited to N-HARQ times. The device starts/restarts a contention resolution timer $(C R T)$ indicating the maximum duration of the random access procedure (presented in subframes) whenever it transmits the Message 3 [6]. The device declares a random-access failure and reverts to Step (1) to retransmit its RA attempt if the contention resolution timer expires. Note that the Message 3 and the Message 4 are used for carrying connection setup signaling messages as well as for contention resolution[11].

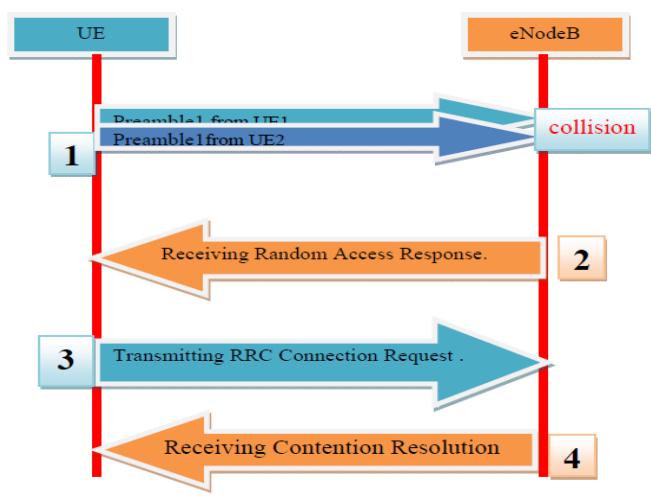

Figure 2: Collision event in Message 1.

\section{SYSTEM SIMULATION AND EVALUATION}

The simulation under consideration of LTE system is built on the following assumptions:

a) Figure 3 shows the simulation model in this paper, includes $500 \mathrm{M} 2 \mathrm{M}$ devices (SDs), one eNB and one server. 
b) The PRACH configuration indexes are set to Format 0. Format 0 demonstrates the small to medium cell size, which covers less than $10 \mathrm{~km}$.

c) The backoff indicator (BI) parameter is applied. The BI will be changed to 20,80 and $160 \mathrm{~ms}$. The best BI parameters for all SDs will be investigated.

d) In the simulation, preamble format 0 ( 1 subframe long), the number of RAR ( $3 \mathrm{~ms})$, the number of CRT $(8,24,48 \mathrm{~ms})$ and HARQ (3 and $5 \mathrm{~ms}$ ) are simulated.

e) All of them are implemented on the suburban zone.

For more parameters, the configuration on the simulation is described in Table 1.

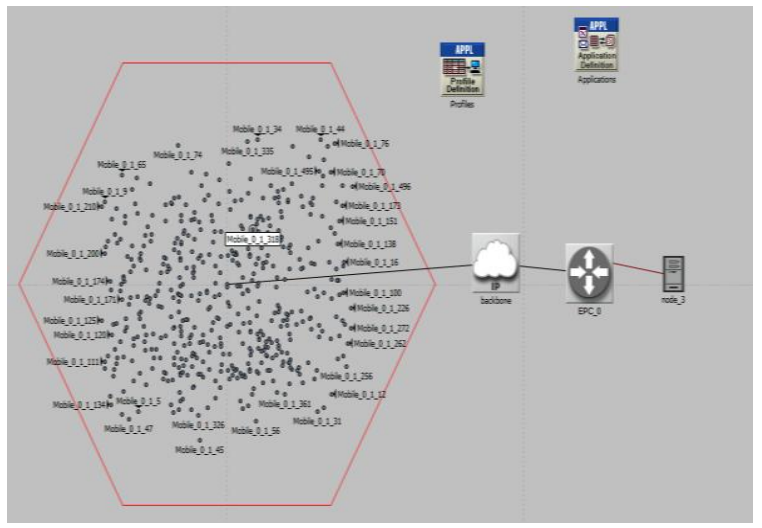

Figure -3 Simulation model.

Table 1:- Parameters And Values

\begin{tabular}{|c|c|}
\hline Parameter & Value \\
\hline Cell bandwidth & $5 \mathrm{MHz}$ FDD \\
\hline PRACH Configuration Index & 6 \\
\hline Preamble Format & Format 0 \\
\hline No. of Preamble & 54 \\
\hline RA-Response Window Size (RAR) & $3 \mathrm{~ms}$ \\
\hline Contention Resolution Timer (CRT) & 8,24 and $48 \mathrm{~ms}$ \\
\hline Backoff Indicator (BI) & 20,80 and $160 \mathrm{~ms}$ \\
\hline $\begin{array}{c}\text { Max. Msg } 3 \text { Retransmission of } \\
\text { HARQ }\end{array}$ & 3,5 \\
\hline Subframe size & $1 \mathrm{~ms}$ \\
\hline RAR Subframe & $5 \mathrm{~ms}$ \\
\hline Path Loss Model & suburban fixed \\
\hline Simulation Time & 600 seconds \\
\hline SM Data Size & 300 bytes \\
\hline PDCCH symbols per sub frame & 1 \\
\hline M2M Traffic model & 500 user \\
\hline Arrival distribution & uniform distribution \\
\hline Distribution period (T) & $60 \mathrm{~s}$ \\
\hline
\end{tabular}

\section{RESULTS AND DISCUSSION}

The results are classified into the three categories:

\subsection{LTE Global Parameters}

Figure 4-a indicates backoff performance at case 1 (CRT $=8$, MSG3=3). When a collision occurs at an eNode-B, the backoff is activated. If $\mathrm{BI}$ is set to 20 , it means that all UEs can send the next random access preamble after $20 \mathrm{~ms}$. Random access (RA) preambles may collide again, with an increasingly negative impact of the delay. If the $\mathrm{BI}$ is set to $(80 \mathrm{~ms})$ and $(160 \mathrm{~ms})$, after RA preambles hit each other, all MDs have to wait a long time [12]. It also causes an increased delay of the random access procedure. It is clear that the BI $20 \mathrm{~ms}$ is the best parameter due to the lowest delay.
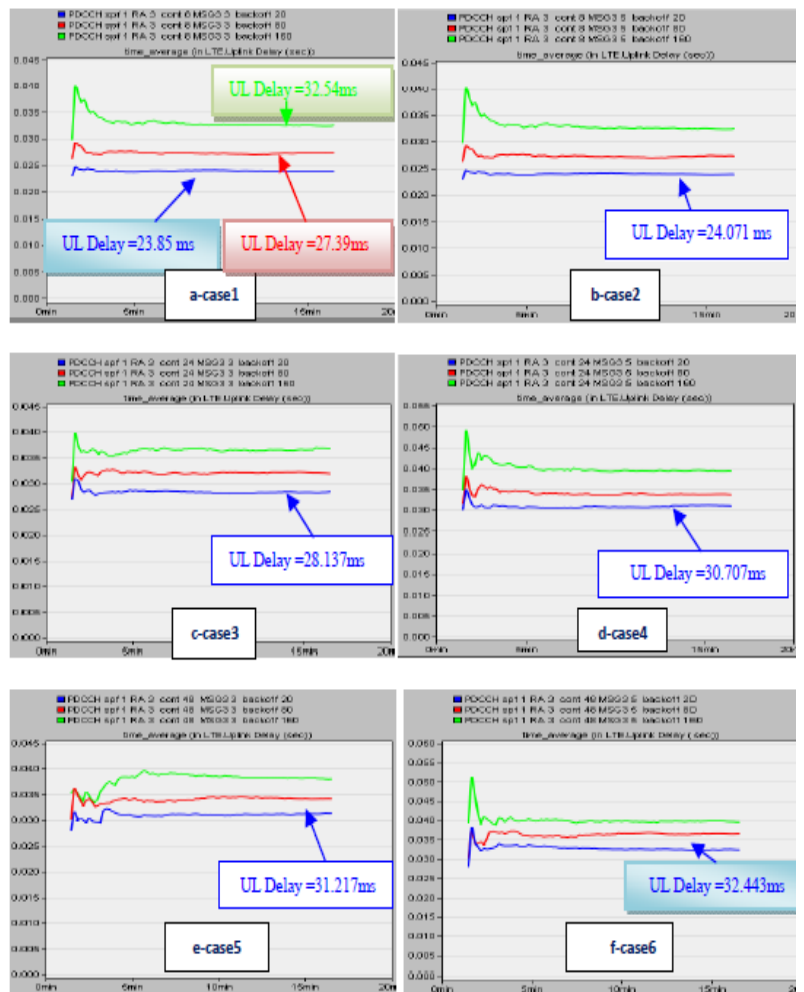

Figure. 4. Uplink Delay

The comparable performance of the uplink delay for different cases when the BI is set to (20ms) is depicted in Table 2 and observed in Figure 4. In the Figure 4-a and Figure 4-f, the average UL delay in case 1 is $23.85 \mathrm{~ms}$ (the minimum delay) and in case $6(\mathrm{CRT}=160 \mathrm{~ms}, \mathrm{MSG} 3=5)$ gives the maximum delay of $32.443 \mathrm{~ms}$ in Uplink. Again UL delay when the BI is set to $(80 \mathrm{~ms})$ for case 1 is $27.391 \mathrm{~ms}$. On the other hand, UL delay when the BI is set to (160ms) for case 1 is $32.54 \mathrm{~ms}$. From the figure $4-\mathrm{a}$, it can be seen that improved UL delay is achieved by choosing the best parameter.

In downlink delay, the performance of all different cases almost remained the same as shown in figure 5. 

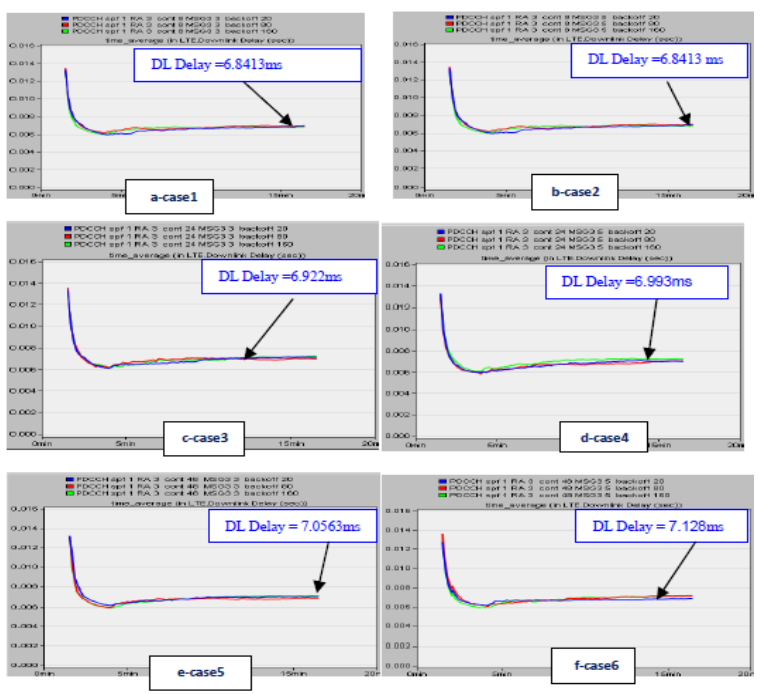

Figure. 5. Downlink Delay

In MD throughput, the performance of all different BI parameter values almost remained the same as illustrated in figure 6 . This is because the $\mathrm{BI}$ require a channel request only. The average MD throughput is approximately 10943 bps in case 1 .
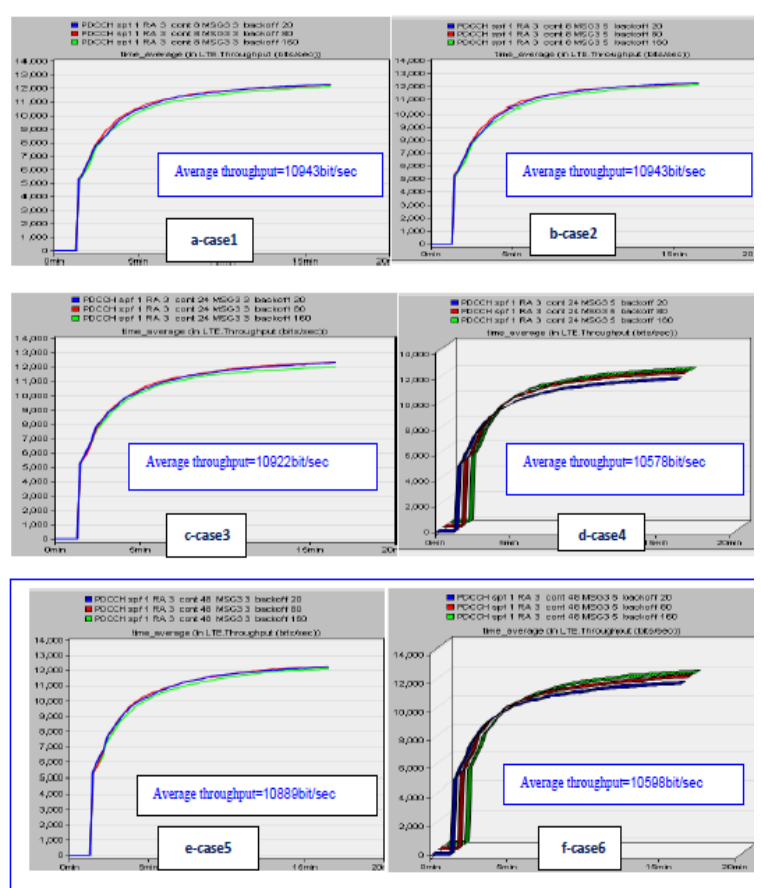

Figure. 6. MD throughput

The comparable performance of the M2M end-to-end delay for different cases when the BI is set to (20ms) is depicted in Table 2 and observed in Figure 7. In the Figure 7-a and Figure 7-f, the average $\boldsymbol{M} \mathbf{2 M} \boldsymbol{E} 2 \boldsymbol{E}$ delay in case1 is $603 \mathrm{~ms}$ (the minimum delay) and in case- 6 gives the maximum $\boldsymbol{M} 2 \boldsymbol{M}$ $\boldsymbol{E} 2 \boldsymbol{E}$ delay is $614 \mathrm{~ms}$. Again $\boldsymbol{M 2 M} \boldsymbol{E} 2 \boldsymbol{E}$ delay when the BI is set to $(80 \mathrm{~ms})$ for case6 is $673 \mathrm{~ms}$. On the other hand, $\boldsymbol{M} \mathbf{M} \boldsymbol{M}$ $\boldsymbol{E} 2 \boldsymbol{E}$ delay when the BI is set to $(160 \mathrm{~ms})$ for case 6 is $609 \mathrm{~ms}$. From the figure 7, it can be seen that improved M2M E2E delay is achieved by choosing the best parameter.

\subsection{LTE PHY Parameters}

The comparable performance of the Time-average(in LTE PHY Uplink packets Dropped (packets/sec)) for different cases when the BI is set to (20ms) is depicted in Table 2 and observed in Figure 8. In the figure 8-a and figure 8-f, the average Packets Dropped (packets/sec) in case1 is 71(packet/sec) (the minimum packet dropped) and in case-6 gives the maximum packets Dropped is 331(packet/sec). Again the average packets Dropped when the BI is set to (80ms) for case6 is 330 (packet/sec). On the other hand, the average packets Dropped when the BI is set to $(160 \mathrm{~ms})$ for case6 is 314(packet/sec). From the figure 8, it is clear that the CRT and MSG. 3 are affected by packets Dropped. The results shown(describe) that if the number of CRT increases, the packets Dropped increases.
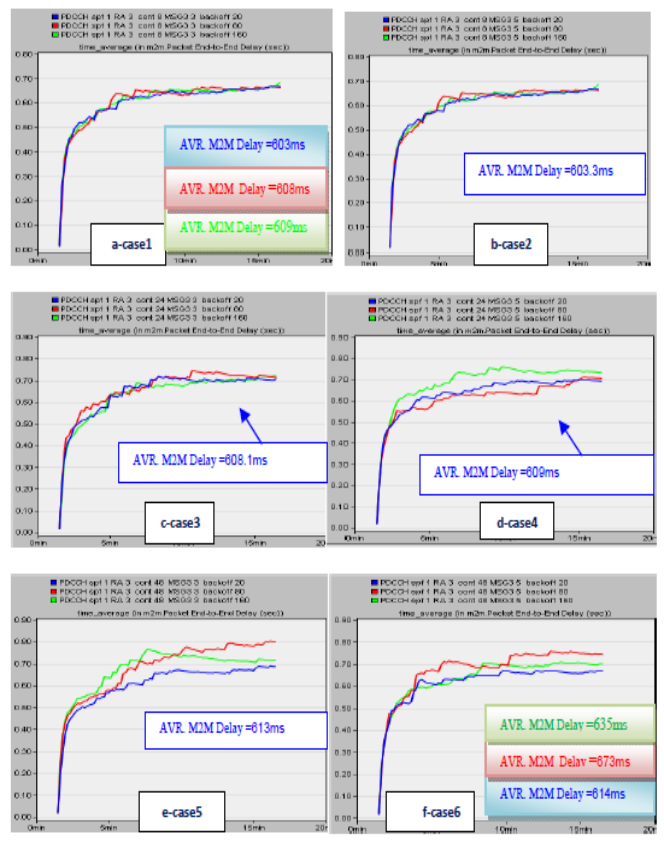

Figure. 7. M2M E2E Delay
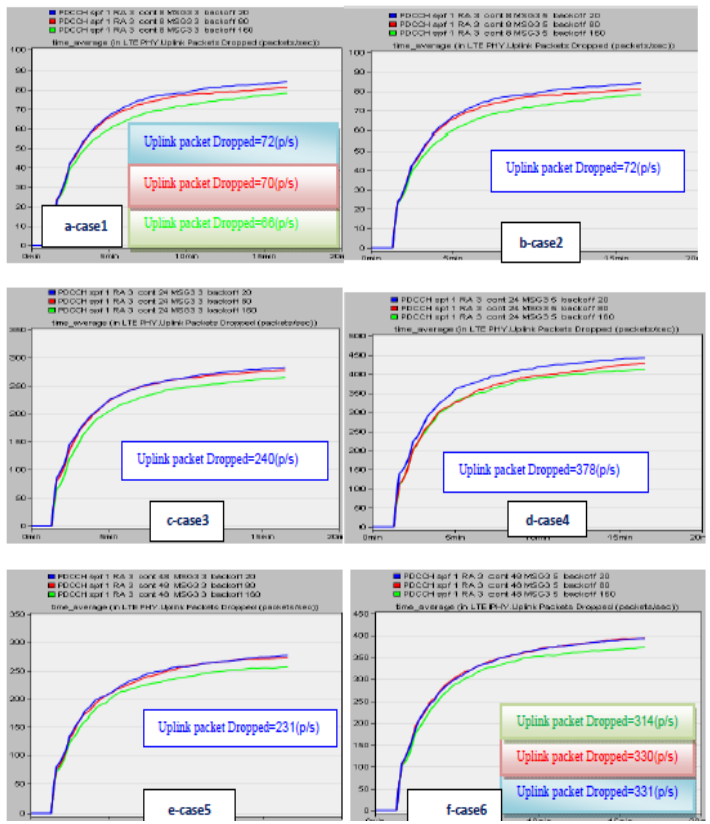

Fig. 8. Time-average(in LTE PHY Uplink packets Dropped (packets/sec)) 
In Downlink packets Dropped (packets/sec), the performance of all different cases almost remained the same as shown in figure 9 .

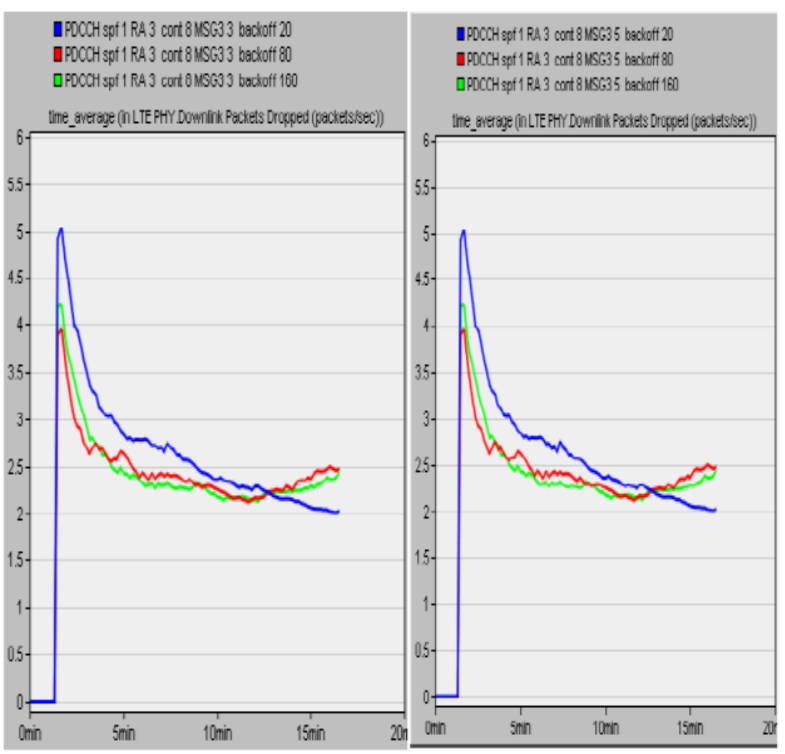

Fig. 9. Time-average(in LTE PHY Downlink packets Dropped (packets/sec))

\subsection{LTE HARQ Parameters}

Retransmission refers to resending the damaged or lost packets in computer network communication. This parameter can be defined as the total number of retransmission attempts by all MACs in a network which may have been damaged or lost due to links failure. This parameter not only determines the rate of retransmission attempt but can also figure out the number of packet drops per second, which has to be retransmitted. The objective of this mechanism is to establish a reliable communication in computer networks. It is virtually identical to the Automatic Repeat request (ARQ). $\mathrm{ARQ}$, an error detection technique consisting of retransmission principles is used to minimize packet transmission errors that take place in the LTE network. An improved version of the ARQ is called Hybrid Automatic Repeat request (HARQ). ARQ lacks error correction if anything goes wrong with the transmitted packets. On the other hand, HARQ is capable of detecting the errors and eventually correcting if transmitted packets are lost on the way to its destination [14].

In all the scenarios of Figure 10, the highest packet drops are observed at the beginning of the transmission. Hence, the maximum retransmissions are attempted at that period. Consequently, packet losses are increased, which enhance the rate of retransmissions [15]. The numbers of retransmission are also increased due to the low number of BI.
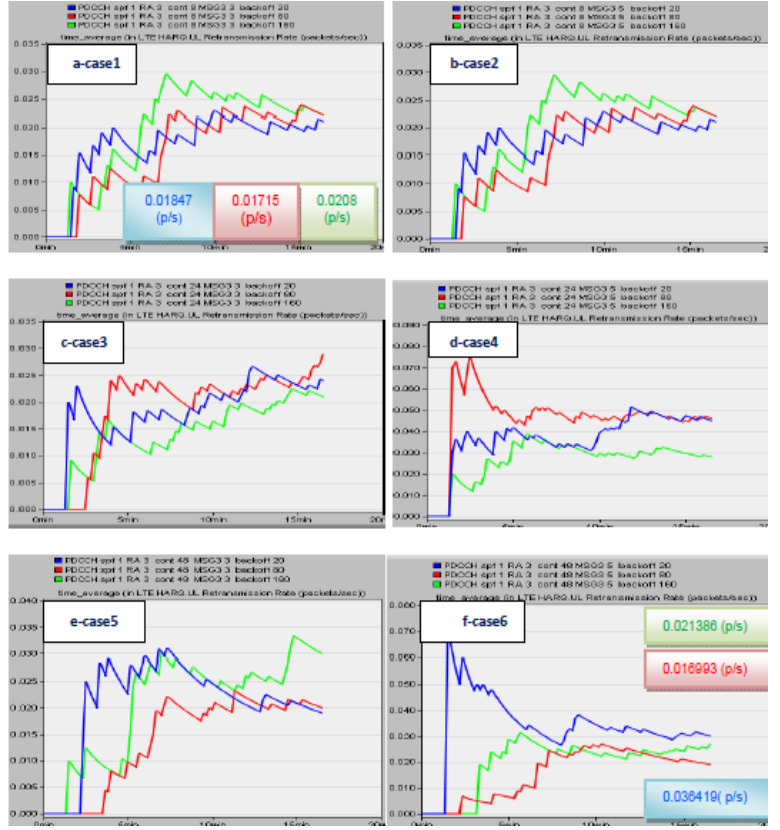

Fig. 10. Time-average(in LTE HARQ UL Retransmission rate (packets/sec))

The goal of this subsection is to evaluate the performance in terms of HARQ DL Retransmission rate for the different cases of RACH procedures. In figure 11 shows that the HARQ DL Retransmission rate.
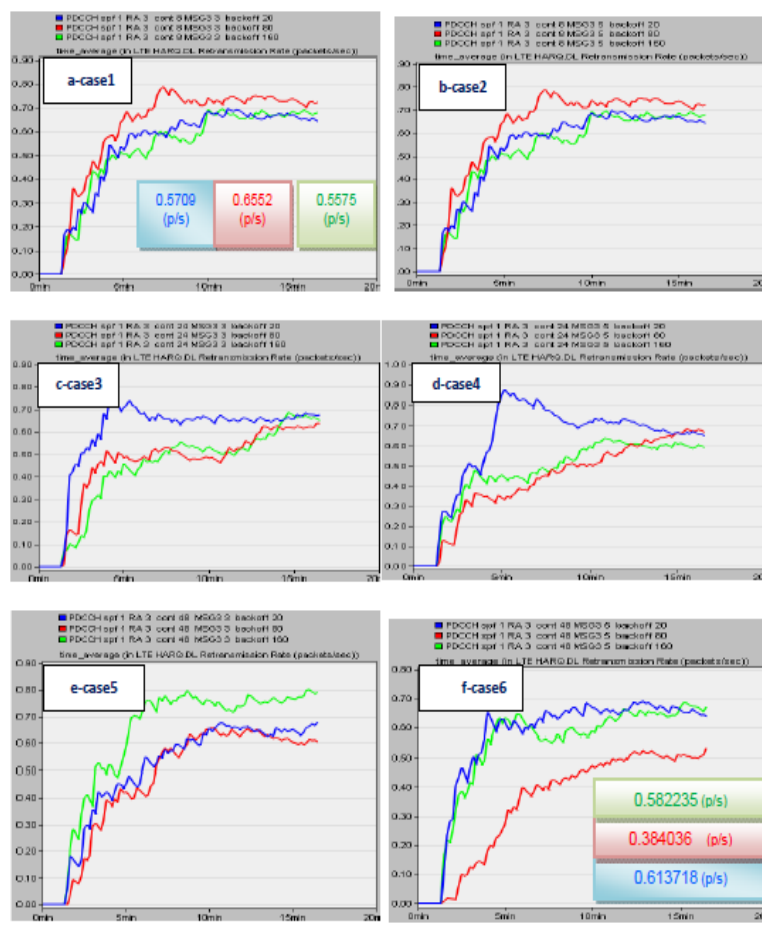

Fig. 11. Time-average(in LTE HARQ DL Retransmission rate (packets/sec))

\subsection{COMPARATIVE ANALYSIS}

The network performance is analyzed in terms of UL/DL delay, throughput, M2M E2E delay, UL/DL packets Dropped, UL/DL Retransmission rate and UL/DL transmission rate. Comparative analysis of each case is summarized below in Table 2: 
Table 2. Comparative analysis of $\mathrm{RACH}$ parameter with respect to LTE's parameters

\begin{tabular}{|c|c|c|c|c|c|c|c|c|c|c|}
\hline $\begin{array}{l}\text { RACH } \\
\text { Parameters } \\
\text { BI= }=20 \mathrm{~ms} \\
\text { RAR }=3 \mathrm{~ms} \\
\end{array}$ & $\begin{array}{l}\text { drerage II2.II } \\
\text { packet E.E. } \\
\text { delary (ms) }\end{array}$ & $\begin{array}{l}\text { drenge } \\
\text { Throughput } \\
\text { (bitSec) }\end{array}$ & \multicolumn{2}{|c|}{$\begin{array}{l}\text { Delay } \\
\text { (ms) }\end{array}$} & \multicolumn{2}{|c|}{$\begin{array}{c}\text { LIE HARQ } \\
\text { Re- } \\
\text { Transmission } \\
\text { Rate (PacketsS) }\end{array}$} & \multicolumn{2}{|c|}{$\begin{array}{c}\text { LIE HRAQ } \\
\text { Iransmission } \\
\text { Rate } \\
\text { (PacketsS) }\end{array}$} & \multicolumn{2}{|c|}{$\begin{array}{l}\text { Dropped } \\
\text { packets Rate } \\
\text { (Packetss) }\end{array}$} \\
\hline & & & DL & UL & DL & UL & DL & UL & $\mathrm{DL}$ & UL \\
\hline $\begin{array}{c}\text { Cass:1 } \\
\text { CRT }=8 \\
\text { MSG3 }=3\end{array}$ & 603 & 10943 & 6.841 & 23.85 & 0.5709 & $\begin{array}{l}0.018 \\
5\end{array}$ & $\begin{array}{l}1.309 \\
3\end{array}$ & $\begin{array}{l}1.4500 \\
8\end{array}$ & $\begin{array}{l}2002 \\
1\end{array}$ & 72 \\
\hline $\begin{array}{c}\text { Cass:? } \\
\text { CRT }=8 \\
\text { MSG3 }=5\end{array}$ & 603.3 & 10943 & 6.841 & 24.07 & $\begin{array}{l}0.57097 \\
1\end{array}$ & $\begin{array}{l}0.018 \\
472\end{array}$ & $\begin{array}{l}1.360 \\
358\end{array}$ & $\begin{array}{l}1.4500 \\
87\end{array}$ & $\begin{array}{l}2.607 \\
6\end{array}$ & 72 \\
\hline $\begin{array}{l}\text { Case.3 } \\
\text { CRT }=2.4 \\
\text { MSG3 }=3\end{array}$ & 608.3 & 10922 & 6.922 & 28.13 & 0.63480 & 0.019 & 1.478 & 1.5073 & 2091 & 240 \\
\hline $\begin{array}{l}\text { Cass-4 } \\
\text { CRT }=24 \\
\text { MSGS }=5\end{array}$ & 609. & 10578 & 6.993 & 30.71 & 0.06572 & 0.039 & 1.679 & 1.7618 & 2265 & 378 \\
\hline $\begin{array}{c}\text { Cass:5 } \\
\text { CRT }=48 \\
\text { MSG3 }=3\end{array}$ & 613 & 10889 & 7.056 & 31.21 & 0.54196 & 0.022 & 1.415 & 1.4945 & 2.434 & 231 \\
\hline $\begin{array}{c}\text { Case-6 } \\
\text { CRT }=48 \\
\text { MSG }=5\end{array}$ & 614 & 10598 & 7.128 & 32.4 & 0.61372 & 0.036 & 1.610 & 1.6784 & 4.300 & 331 \\
\hline
\end{tabular}

\section{FUTURE WORK AND CONCLUSION}

This paper dealt with the performance of LTE-based M2M Communication system and studied the effect of RACH procedure on the performance of the system. It is analyzed in terms of UL/DL delay, throughput, M2M E2E delay, UL/DL packets Dropped, UL/DL Retransmission rate and UL/DL transmission rate as given in Table 2 . It has been observed that performance is varying according to the different cases of $\mathrm{RACH}$ parameters. Case 1 when $(\mathrm{BI}=20 \mathrm{~ms}$, RAR $=3 \mathrm{~ms}$, $\mathrm{CRT}=8 \mathrm{~ms}, \mathrm{MSG} 3=3 \mathrm{~ms}$ ) achieved the best result in terms of the delay, throughput and packets Dropped. The results show that the performance of the system is improved after deploy $\mathrm{RACH}$ procedure. It is noted from this results, that the effect of RACH procedure on the performance of the system is clearly decreased at Case 6 when $(B I=160 \mathrm{~ms}$, RAR=3ms, $\mathrm{CRT}=48 \mathrm{~ms}, \mathrm{MSG} 3=5 \mathrm{~ms}$ ).

For future work, we plan to extend the simulation for the new $\mathrm{RACH}$ procedure which would comprise all the advantage of each RACH parameter.

\section{REFERENCES}

[1] T. Sauter, M. Lobashov " End-to-End Communication Architecture for Smart Grids". IEEE Transactions on Industrial Electronics. http://dx.doi.org/10.1109/TIE.2010.2070771.

[2] C. Karupongsiri, K. S. Munasinghe, and A. Jamalipour, "Random Access Issues for Smart Grid Communication in LTE Networks", IEEE Magazine, 2014.

[3] M. Cheng, G. Lin, and H. Wei, "Overload Control for Machine-Type-Communications in LTE-Advance
System", IEEE Communication Magazine”, pp.38-45, 2012.

[4] M. Hasan, and E. Hossain, "Random Access for Machine-to-Machine Communication in LTE-Advanced Network: Issues and Approaches", IEEE Magazine, pp.86-93, 2013.

[5] D. T. Wiriaatmadja, and K. W. Choi, "Hybrid Random Access and Data Transmission Protocol for Machine-toMachine Communication in Cellular Networks", IEEE Transactions on Wireless Communications", pp.1-14, 2013.

[6] C. H. Wei, R. G. Cheng and S. L. Tsao, "Performance Analysis of Machine-Type Communications in LTE Networks, " IEEE Transactions Technology, vol. 62, no. 7, pp. 3371-3382, Sept. 2013.

[7] A. Laya, L. Alonso, and J. Alonso-Zarate, "Contention Resolution Queues for Massive Machine Type Communications in LTE," IEEE Personal, Indoor, and Mobile Radio Communications (PIMRC)Workshop on Machine-to-Machine Communications, pp. 2314-2318, Hong Kong, 2015.

[8] 3GPP TS 36.325, "Evolved universal terrestrial radio access Medium access control (MAC) protocol specification," Third-Gen. Partnership Proj, SophiaAntipolis Cedex, France, ver. 9.3.0, Jun. 2010.

[9] IEEE 802.16m-08/413, "Synchronous Non-adaptive HARQ in IEEE 802.16m Uplink”.

[10] Yang Ping," Distributed Queuing-based Random Access Procedure in Mobile Networks", Master thesis, May 2016.

[11] A. Laya, L. Alonso, and J. Alonso-Zarate, "Contention Resolution Queues for Massive Machine Type Communications in LTE," IEEE Personal, Indoor, and Mobile Radio Communications (PIMRC)Workshop on Machine-to-Machine Communications, pp. 2314-2318, Hong Kong, 2015

[12] 3GPP R2-100182, "Access control of MTC devices," CATT, RAN2\#68bis, Valencia, Spain, Jan. 2010.

[13] 3GPP, TS 36.321 ver. 11.2.0, Rel.11, "Evolved Universal Terrestrial Radio Access (E-UTRA); Medium Access Control and Protocol Specification," Technical Specification, 2013.

[14] G. A. A., M. I., and K. Jumari, "Behaviour of CWND for Different TCP over Parameters of LTE Networks," I T Journal, ISSN: 1812-5638, Science Alert, 2010.

[15] W. R. Stevens, "TCP slow start, congestion avoidance, fast retransmit, and fast recovery algorithms," IETF RFC 2001, 2001.

[16] A. Laya, L. Alonso, and J. Alonso-Zarate, "Is the Random Access Channel of LTE / LTE-A Suitable for M2M Communications? A Survey of Alternatives," IEEE, vol. 16, no. 1, pp. 4-16, 2014. 